\title{
Population Pharmacokinetics And Bayesian Estimation of Mycophenolate Mofetil In Patients With Autoimmune Hepatitis
}

\author{
Tom Nanga $^{1}$, Jean-Baptiste Woillard ${ }^{1}$, Annick Rousseau ${ }^{1}$, Pierre Marquet ${ }^{1}$, and Aurélie \\ Prémaud $^{1}$ \\ ${ }^{1}$ Limoges University
}

November 1, 2021

\begin{abstract}
Background: Mycophenolate mofetil (MMF) is the most widely used second-line agent in auto-immune hepatitis (AIH). It is generally titrated up to patient response and continued for at least two years following complete liver enzyme normalization. However, in this maintenance phase individual dose adjustment to reach mycophenolic acid (MPA) exposure with the best benefit-risk probability may avoid adverse outcomes. The aim of the present study was to develop population pharmacokinetic (popPK) models and Maximum A-Posteriori Bayesian estimators (MAP-BEs) to estimate MPA inter-dose area under the curve (AUC0-12h) in AIH patients administered MMF using nonlinear mixed effect modelling. Methods: We analysed 50 MPA PK profiles from 34 different patients, together with some demographic, clinical, and laboratory test data. The median number of samples per profile, immediately preceding and following the morning MMF dose, was 7 [4-10]. PopPK modeling was performed using parametric, top-down, nonlinear mixed effect modelling with NONMEM 7.3. MAP-BEs were developed based on the the best popPK model and the best limited sampling strategy (LSS) selected among several. Results: The pharmacokinetic data were best described by a 2-compartment model, Erlang distribution to describe the absorption phase, and a proportional error. The best MAP-BE relied on the LSS at $0.33,1$ and 3 hours after mycophenolate mofetil dose administration and was very accurate (bias $=5.6 \%$ ) and precise $(\mathrm{RMSE}<20 \%)$. Conclusion: The precise and accurate Bayesian estimator developed in this study for AIH patients on MMF can be used to improve the therapeutic management of these patients.
\end{abstract}

\section{Hosted file}

Article_MPA__submitted.docx available at https://authorea.com/users/444018/articles/543868population-pharmacokinetics-and-bayesian-estimation-of-mycophenolate-mofetil-inpatients-with-autoimmune-hepatitis 


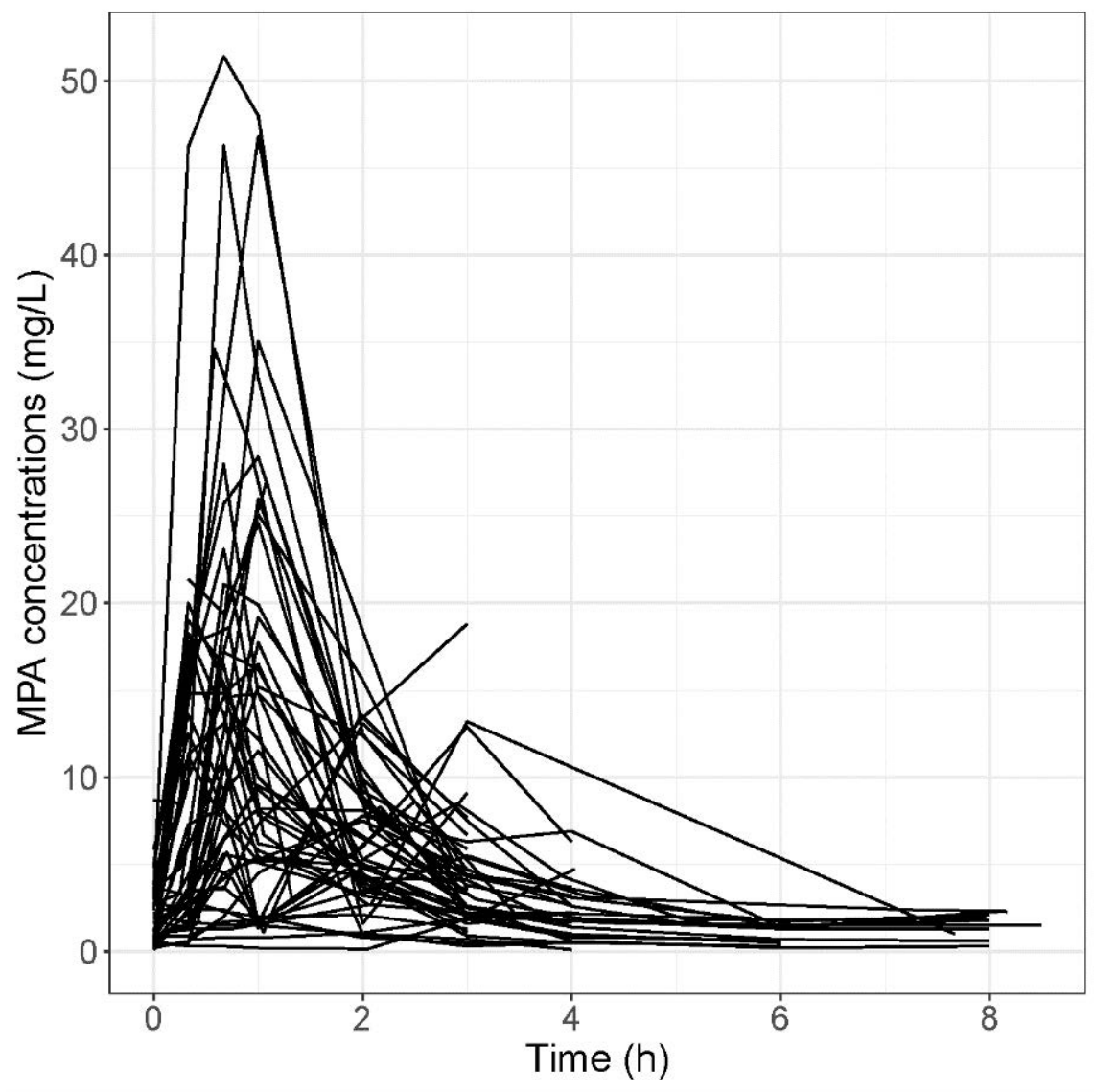



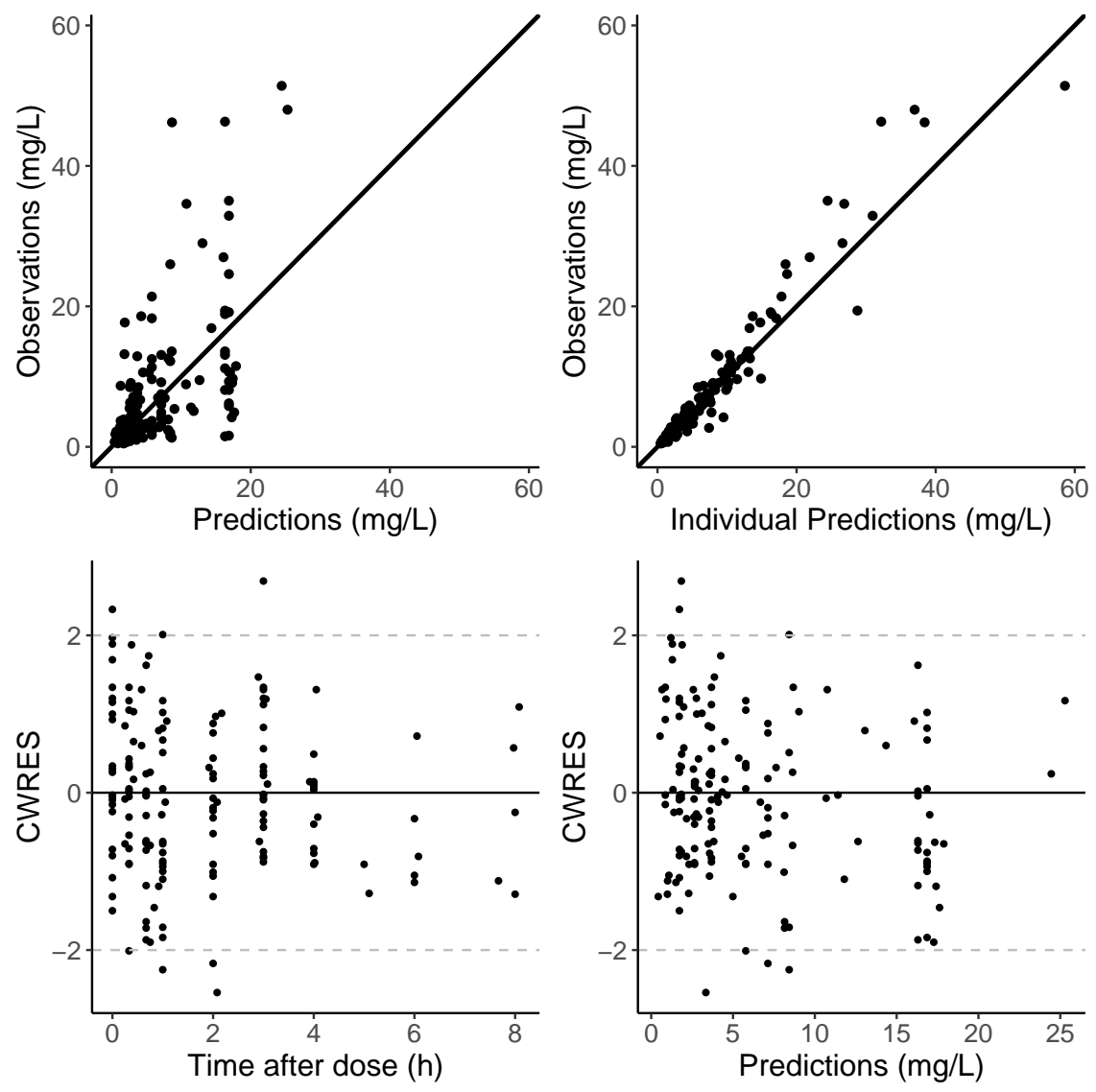


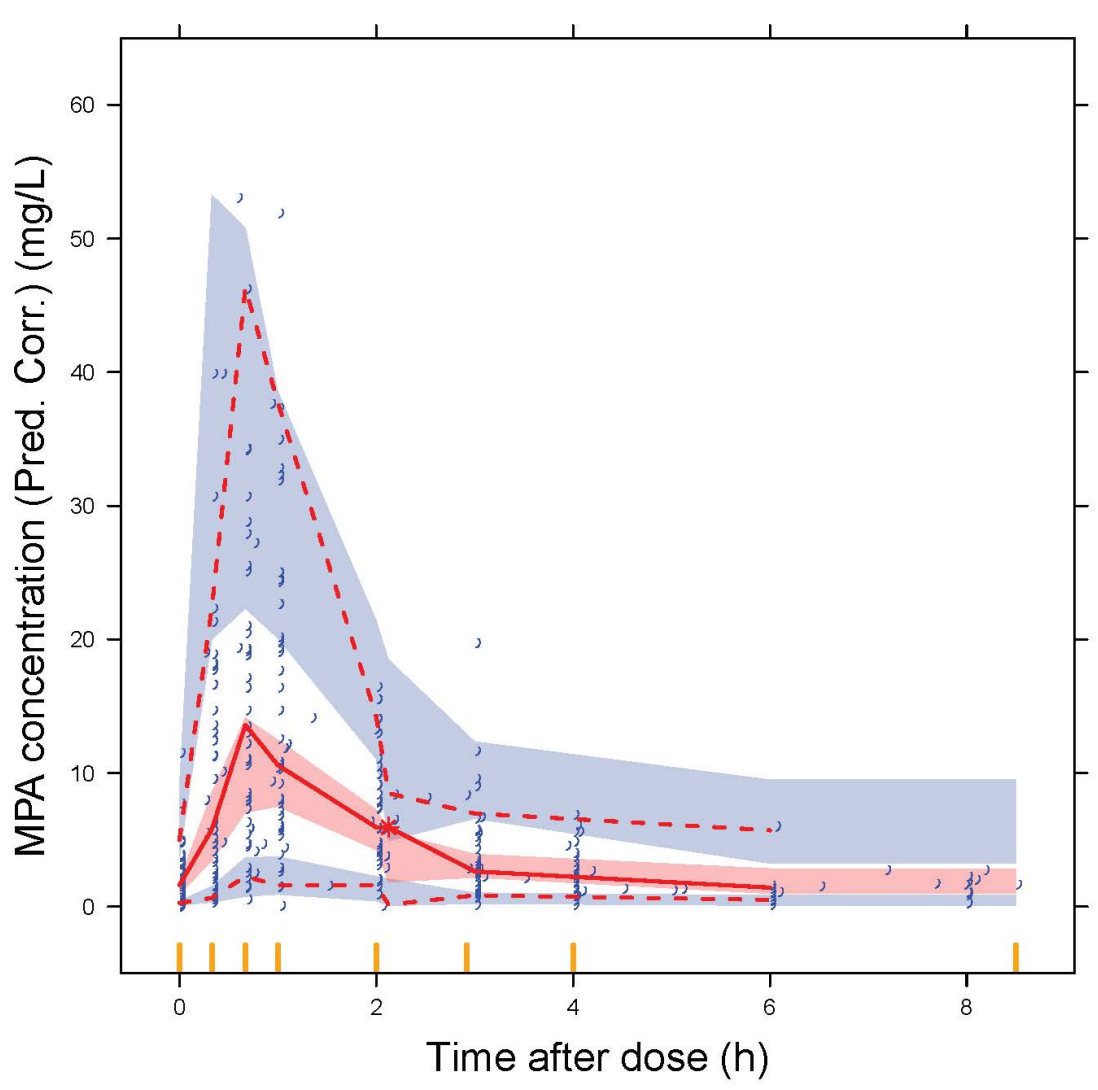

